\title{
Notch-induced Asb2 expression promotes protein ubiquitination by forming non-canonical E3 ligase complexes
}

\author{
Lei Nie ${ }^{1}$, Ying Zhao ${ }^{1}$, Wei Wu${ }^{1}$, Yuan-Zheng Yang ${ }^{1}$, Hong-Cheng Wang ${ }^{1}$, Xiao-Hong Sun ${ }^{1}$ \\ ${ }^{I}$ Immunobiology and Cancer Research Program, Oklahoma Medical Research Foundation, 825 NE 13th Street, Oklahoma City, \\ OK 73104, USA
}

\begin{abstract}
Notch signaling controls multiple developmental processes, thus demanding versatile functions. We have previously shown that this may be partly achieved by accelerating ubiquitin-mediated degradation of important regulators of differentiation. However, the underlying mechanism was unknown. We now find that Notch signaling transcriptionally activates the gene encoding ankyrin-repeat SOCS box-containing protein 2 (Asb2). Asb2 promotes the ubiquitination of Notch targets such as E2A and Janus kinase (Jak) 2, and a dominant-negative (DN) mutant of Asb2 blocks Notch-induced degradation of these proteins. Asb2 likely binds Jak2 directly but associates with E2A through Skp2. We next provide evidence to suggest that Asb2 bridges the formation of non-canonical cullin-based complexes through interaction with not only Elongin B/C and Cullin (Cul) 5, but also the F-box-containing protein, Skp2, which is known to associate with Skp1 and Cul1. Consistently, ablating the function of Cul1 or Cul5 using DN mutants or siRNAs protected both E2A and Jak2 from Asb2-mediated or Notch-induced degradation. By shifting monomeric E3 ligase complexes to dimeric forms through activation of Asb2 transcription, Notch could effectively control the turnover of a variety of substrates and it exerts diverse effects on cell proliferation and differentiation.
\end{abstract}

Keywords: Notch; Asb2; ubiquitin; Jak2; Skp2

Cell Research (2011) 21:754-769. doi:10.1038/cr.2010.165; published online 30 November 2010

\section{Introduction}

The Notch signaling pathway is an evolutionarily conserved pathway that controls vital processes in the development of organisms ranging from Drosophila to humans $[1,2]$. Activation of the pathway is triggered by ligation of Notch receptors and their ligands Jagged or Delta-like, which leads to the proteolytic cleavage of the receptors thereby releasing the intracellular domain (IC). The IC then translocates into the nucleus and activates transcription by interacting with its DNA-binding partner, CSL, and recruiting transcriptional co-activators such as $\mathrm{p} 300$ and mastermind [3-5]. A large number of genes have been found to be regulated by Notch signaling. However,

Correspondence: Xiao-Hong Sun

Tel: +405-271-7103; Fax: +405-271-7128

E-mail: sunx@omrf.org

Received 3 August 2010; revised 11 October 2010; accepted 20 October 2010; published online 30 November 2010 considering the diversity of cellular differentiation programs regulated by Notch, it is likely that Notch employs global control mechanisms. We have previously shown that activation of Notch signaling pathways accelerates ubiquitin-mediated degradation of several important regulators of differentiation, namely, E2A and SCL/Tal1 transcription factors, as well as the Janus kinase (Jak) 2 and Jak3 [6-8]. Notch-induced degradation of these proteins is MAP kinase-dependent and thus occurs in a cell type-specific manner $[6,7]$. As both E2A (including two alternatively spliced variants, E12 and E47, which differ only in the DNA-binding and dimerization domains) and Jak proteins play crucial roles in lymphocyte development, their degradation induced by Notch signaling may contribute to suppression of B-cell differentiation. In contrast, E2A and Jak2 degradation does not occur in T cells, thus allowing Notch to promote T-cell development $[7,9]$. However, the effect of Notch may not be restricted solely to the ubiquitination substrates mentioned above. A better understanding of the molecular mechanism by 
which Notch mediates the degradation of these proteins will help identify additional targets and determine the importance of Notch-induced protein turnover in various aspects of developmental biology.

Our previous studies demonstrated that Notch-induced ubiquitination of $\mathrm{E} 2 \mathrm{~A}$ proteins was catalyzed by the $\mathrm{SCF}^{\text {Skp2 }}$ E3 ligase complex containing Skp1, Cullin 1 (Cul1) and F-box protein, Skp2, which functions as the substrate-binding subunit $[6,8,10,11]$. In contrast, Jak2 has been shown to be ubiquitinated by the ECS ligase complex consisting of ElonginB, ElonginC, Cullin 5 (Cul5) and SOCS [12, 13]. SOCS proteins bind Jak2 via their SH2 domain and contact ElonginC and $\mathrm{Cul} 5$ with the SOCS box [14]. Both SCF ${ }^{\text {Skp2 }}$ and ECS complexes belong to the cullin-ring family of E3 ligases [15]. Cul1 and Cul5 are thought to serve as scaffolds by interacting at their $\mathrm{N}$-termini with their respective adaptors such as Skp1 and ElonginB/C, which then engage substratebinding subunits such as Skp2 and SOCS. The C termini of cullins are bound to ring-finger proteins such as Rbx 1 or 2, which recruit the E2 ubiquitin-conjugating enzyme that is responsible for transferring the ubiquitin moiety to substrates [16]. Neddylation at the C terminus of cullins plays an important role in regulating the E3 ubiquitin ligase activity by facilitating the proper orientation of Rbx and preventing their association with an inhibitory molecule, CAND1 [17-20]. Despite the similarities in the general structure and function of these two E3 ligase complexes, $\mathrm{SCF}^{\mathrm{Skp} 2}$ and ECS have non-overlapping components and substrates. Therefore, a critical question that remains to be answered is how Notch signaling enhances the activity of these two, very different E3 ligases.

We also showed that Notch-mediated transcriptional events were required for degradation of the target proteins [6]. Since we did not detect any significant alterations in the levels of the known components of $\mathrm{SCF}^{\mathrm{Skp} 2}$ and ECS ligases upon Notch signaling, we performed a microarray analysis to search for novel target genes that might be responsible for stimulating the activities of these ligases. One of the genes identified codes for the ankyrin-repeat SOCS box-containing protein 2 (Asb2) [21]. This protein belongs to a family with 18 members, Asb1-18, which all possess a SOCS box and varying numbers of ankyrin repeats [14]. A few members of this family have been shown to promote protein degradation but the mechanism of action is not known $[22,23]$. Asb2, as well as several other Asb proteins, have been shown to interact with ElonginB/C and Cul5 [21, 24, 25]. Since Jak2 ubiquitination is known to involve Elongin and Cul5 [12, 26], it is conceivable that Asb2 could facilitate the ubiquitination of Jak proteins. However, whether or not Asb2 has a role in the ubiquitination of substrates of the $\mathrm{SCF}^{\mathrm{Skp} 2}$ complex such as E2A proteins remained unknown.

In this report, we demonstrate that Asb2 serves as a mediator of Notch to promote the ubiquitination of both E47 and Jak2. Furthermore, we present evidence to suggest a possible mechanism whereby Asb2 facilitates the ubiquitination of these proteins known to be modified by different E3 ligases, thus explaining how Notch signaling can trigger the turnover of a wide array of substrates and exert diverse biological activities.

\section{Results}

Notch signaling stimulates Asb2 expression to promote protein degradation

Our previous studies revealed that Notch-induced E47 degradation depended on its ability to transcriptionally activate gene expression through interaction with its DNA-binding partner, CSL [6]. Hence, it is likely that Notch signaling turns on genes involved in protein ubiquitination and degradation. Although Skp2 was thought to be controlled by Notch signaling [27, 28], we found Notch signaling did not result in more than two-fold alterations in mRNA levels of any components of $\mathrm{SCF}^{\text {Skp2 }}$ in several cell types (data not shown). Next, we performed microarray analyses using Affimetrix chips and RNA isolated from BaF3 hematopoietic cells transduced with retrovirus expressing the IC of Notch1 (N1-IC), which is the constitutively active form of the receptor. Although over 1000 genes were found to be upregulated by N1-IC, only a few of them were known to be involved in protein ubiquitination. Among these, Asb2 was stimulated 26-fold by N1-IC compared with the vector control. This finding was then verified by using real-time RTPCR assays, which showed that N1-IC increased Asb2 mRNA levels by an average of 66 -fold in $\mathrm{BaF} 3$ cells (Figure 1A). Correspondingly, the level of Asb2 protein was also markedly elevated (Figure 1A).

Next, we examined Asb2 expression in primary pre-B cells, where activation of Notch signaling pathways accelerates the degradation of both E2A and Jak2/3 proteins [7]. Mouse bone marrow cells were first depleted of myeloid, erythroid and $\mathrm{T}$ lineage cells plus mature B cells, resulting in a population consisting of predominantly pre-B cells and a small fraction of various hematopoietic progenitors. To activate endogenous Notch receptors, these cells were then co-cultured with stromal cells transduced with a retrovirus expressing a Notch ligand, Delta-like-1 (DL-1). As shown in Figure 1B, exposure to the Notch ligand led to a 42-fold increase in the level of Asb2 mRNA compared with cells co-cultured on control stromal cells, with accompanying increases in the 

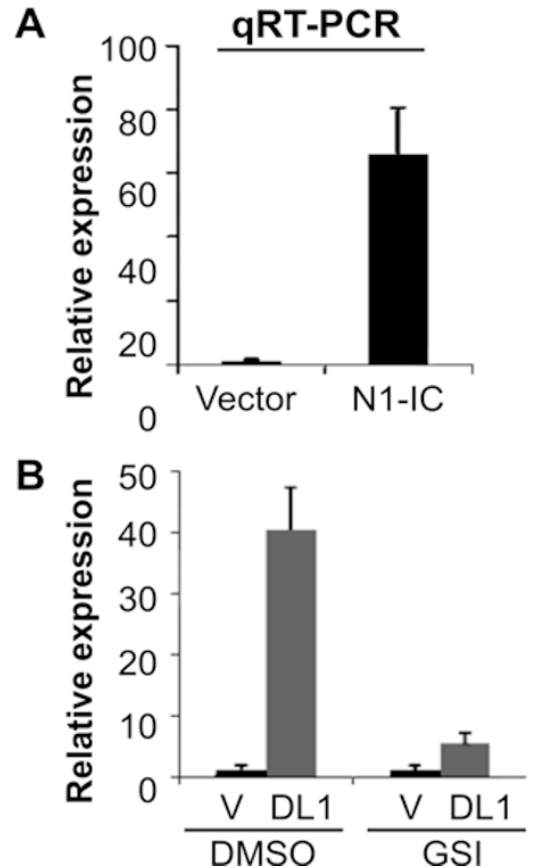
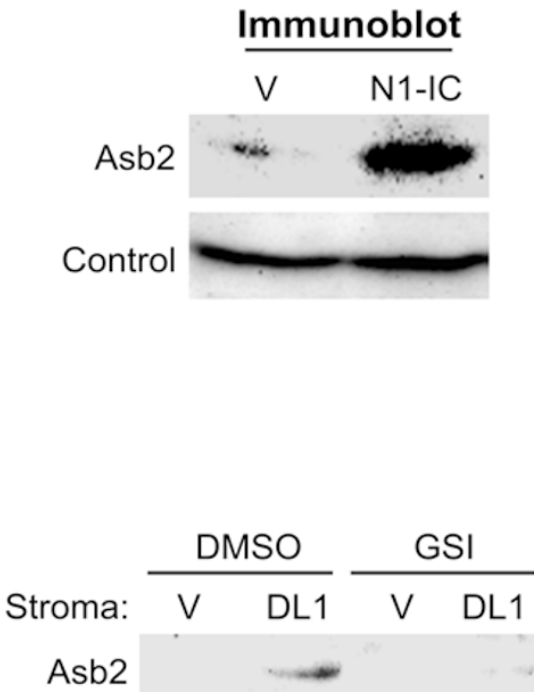

Control

Figure 1 Notch signaling upregulates Asb2 expression. (A) BaF3 cells were transduced with retrovirus expressing GFP or GFP plus the IC of Notch1 (N1-IC). GFP-positive cells were sorted and used for RNA isolation and quantitative real-time PCR (qRT-PCR). Levels of transcripts were normalized against that of $\beta$-actin by calculating $\Delta \mathrm{C}_{\mathrm{T}}$. Data shows the average $\pm \mathrm{SD}$, which was calculated as described [51], and is a representative of three independent experiments. For immunoblotting, total lysates of the same cells were used with antibodies against Asb2 and tubulin as a loading control. (B) Lin $^{-} \mathrm{CD} 19^{+} \operatorname{lgM}{ }^{-}$bone marrow cells prepared by immune-magnetic depletion with antibodies against Mac-1, Ter119, CD3, CD8 and IgM were cocultured on OP42 stromal cells transduced with vector or DL1-expressing retrovirus in the presence of cytokines (10 $\mathrm{ng} / \mathrm{ml}$ SCF, $10 \mathrm{ng} / \mathrm{ml} \mathrm{Flt-3} \mathrm{ligand} \mathrm{and} 5 \mathrm{ng} / \mathrm{ml} \mathrm{IL-7)}$ ) along with $10 \mu \mathrm{M}$ of a GSI or DMSO for $1.5 \mathrm{~h}$. The co-cultured bone marrow cells were carefully harvested and used for qRT-PCR and immunoblot assays as described in $\mathbf{A}$.

level of Asb2 protein. In contrast, addition of a gammasecretase inhibitor (GSI), that blocks Notch activation, abolished the effect of DL-1 on Asb2 expression at both mRNA and protein levels (Figure 1B). Thus, these results suggest that Notch activation indeed significantly stimulates Asb2 expression. Our preliminary studies also reveal that Notch signaling could stimulate expression of several additional members of the Asb family in different cell types (data not shown).

\section{Asb2 mediates Notch-induced ubiquitination and degra- dation of E47 and Jak2}

To evaluate the ability of Asb2 to mediate Notch-induced ubiquitination and degradation, we initially chose to examine the effect of Asb2 on the turnover of two representative substrates, E47 and Jak2, ubiquitination of which is thought to be catalyzed by $\mathrm{SCF}^{\text {Skp2 }}$ and ESC E3 ligases, respectively. We transduced $\mathrm{BaF} 3$ cells with retroviruses expressing either Asb2 or N1-IC and compared their effects on the stability of endogenous E47 and Jak2 by immunoblotting (Figure 2A). Asb2 was found to be as effective as N1-IC at diminishing the levels of E47 and Jak2. Moreover, the effect of Asb2 on E47 and Jak2 degradation can be alleviated by treating the cells with a proteasome inhibitor, MG132 (Figure 2A). Consistent with the effect of Asb2 on protein turnover, levels of ubiquitinated endogenous E47 and Jak2 were increased by either Asb2 or N1-IC (Figure 2B). Since Asb2 belongs to a family of 18 members, we asked if other Asb members have similar properties. In co-transfection assays, we found that Asb1 was as efficient as Asb2 in stimulating the degradation of E47 and Jak2 whereas Asb12 failed to do so (Figure 2C). Likewise, Asb2 $\Delta \mathrm{C}$, a deletion mutant lacking the SOCS box, was also unable to promote E47 or Jak2 degradation. These results suggest that while some of the Asb proteins have redundant functions, not all Asb proteins behave similarly.

To determine whether Asb2 mediates Notch-induced protein degradation, we tested the effect of Asb2 downregulation on E47 and Jak2 turnover. We utilized a dominant-negative (DN) mutant, Asb $2 \Delta \mathrm{C}$, to interfere with the function of not only Asb2 but also other Asb proteins 
A
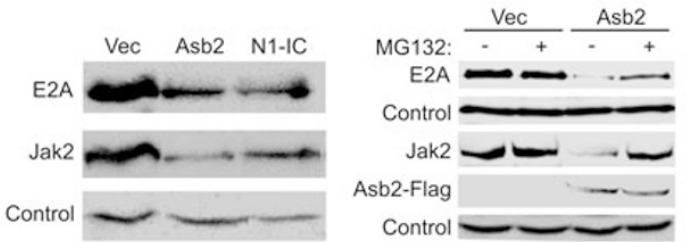

B

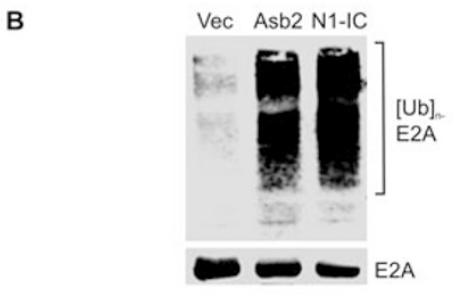

C

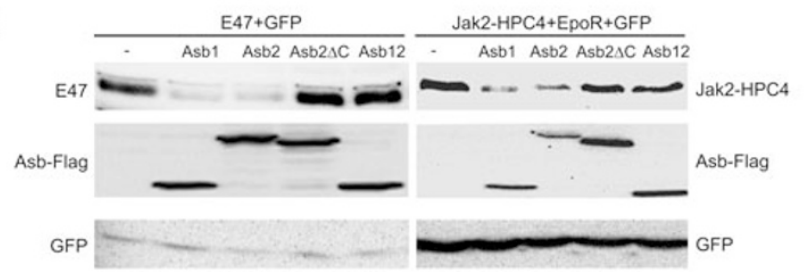

D

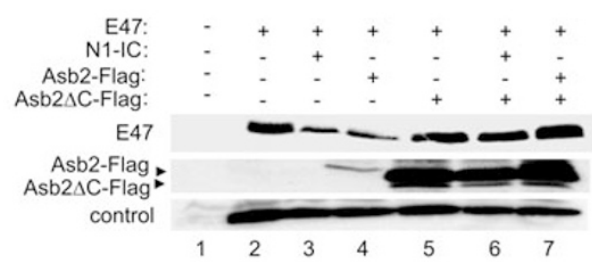

E

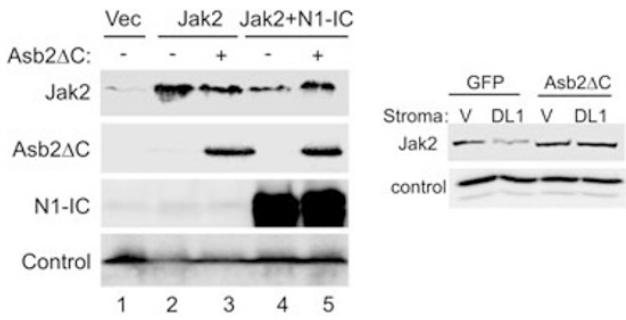

Figure 2 Asb2 mediates Notch-induced E47 and Jak2 degradation. (A) Asb2 accelerates E2A and Jak2 degradation as efficiently as N1-IC. Left, BaF3 cells transduced with vector, HPC4-tagged Asb2 or N1-IC-expressing retroviruses were used for immunoblotting endogenous E2A, Jak2 and tubulin. Right, vector or Asb2-Flag-transduced cells were also treated with 12 $\mathrm{MM}$ MG-132 (+) or DMSO (-) for $2 \mathrm{~h}$. Immunoblotting was performed with antibodies against E2A and Jak2, as well as Erk1 for loading control. Asb2 was detected with an antibody against its HPC4 tag. (B) Stimulation of ubiquitination of E2A and Jak2 by Asb2 in vivo. BaF3 cells transduced with vector, Asb2 and N1-IC retroviruses were treated with $5 \mu \mathrm{M}$ MG-132 for 5 h. Whole-cell extracts were used for IP with anti-E2A and Jak2 antibodies, respectively. The precipitates were immunoblotted with a monoclonal antibody against ubiquitin. The amounts of endogenous E2A and Jak2 proteins brought down were determined by probing with anti-E2A or Jak2 antibodies. (C) Asb1 but not Asb12 induces E47 and Jak2 degradation. Left, E47 plus or minus indicated that Asb constructs were co-transfected into NIH3T3 cells. A plasmid expressing GFP was included in all transfection experiments to monitor transfection efficiency. Right, Jak2 and Epo receptor plus or minus indicated that Asb constructs were co-transfected into $293 \mathrm{~T}$ cells and treated with $5 \mathrm{ng} / \mathrm{ml}$ of Epo for Jak2 activation for $1.5 \mathrm{~h}$ prior to harvest. Immunoblot analyses were performed with antibodies against the indicated proteins. Asb proteins were detected with an anti-Flag antibody whereas control was carried out with anti-GFP antibodies. (D) Notch or Asb2-induced E47 degradation is blocked by a DN mutant of Asb2 (Asb2 $\Delta \mathrm{C}$ ). E47 plus or minus N1-IC or flag-tagged Asb2 were co-transfected into NIH3T3 cells along with or without flag-tagged Asb2 $\Delta$ C. A plasmid expressing GFP was included in all transfection experiments to monitor transfection efficiency. Immunoblot analyses were performed with antibodies against the indicated proteins. (E) Left, Jak2 and Epo receptor plus or minus N1-IC were co-transfected into 293T cells along with or without Asb2 $\Delta \mathrm{C}$ and treated with $5 \mathrm{ng} / \mathrm{ml}$ of Epo for Jak2 activation for $1.5 \mathrm{~h}$ prior to harvest for immunoblotting. Right, BaF3 cells transduced with vector or Asb2 $\Delta$ C-expressing retrovirus were co-cultured with OP42 stromal cells transduced with vector control or retrovirus expressing Notch ligand, DL1, for $1.5 \mathrm{~h}$. Endogenous Jak2 levels were determined by immunoblotting and loading controls were performed by probing tubulin.

with redundant functions. Asb $2 \Delta \mathrm{C}$ has minimal effects on E47 by itself (lanes 2 vs 5, Figure 2D), but significantly rescued N1-IC- or Asb2-induced degradation (lanes 3 vs 6 or 4 vs 7, Figure 2D). Similarly, Notch-induced Jak2 turnover was also blocked by Asb $2 \Delta \mathrm{C}$ not only in transient transfection assays (left, Figure 2E) but also in the BaF3 cells, in which endogenous Notch signaling was triggered by its ligand, DL1, expressed on stromal cells (right, Figure 2E). These results thus suggest that Notch-induced Asb2 expression mediates its effect on the stability of E47 and Jak2.

\section{Asb2 associates with a subset of cullin proteins}

Both E47 and Jak2 are believed to be ubiquitinated by cullin-based E3 ligases raising the question whether Asb2 interacts with any or specific cullin proteins. We therefore performed co-immunoprecipitation (IP) assays by transfecting Flag-tagged Asb2 into 293T cells and examined the ability of the anti-Flag antibody to pull down endogenous Cullin proteins. Input controls of transfected 
and endogenous proteins were immunoblotted in parallel with the immunoprecipitates. Therefore, the relative affinity of Asb2 to each of the cullins could be estimated. Cull, which is known to be involved in Notch-induced ubiquitination of E47, co-precipitated with Asb2 (Figure 3A). Likewise, Cul5 also associated with Asb2 (Figure $3 \mathrm{~A}$ ). Both these interactions were further verified by IP with antibodies against $\mathrm{Cul1}$ or $\mathrm{Cul5}$, followed by immunoblotting for Asb2 (Figure 3B). Although Cul2 and Cul5 are structurally similar, endogenous Cul2 was not brought down with Asb2 (Figure 3A). However, when both Asb2 and Cul2 were overexpressed, Cul2 coprecipitated with $A s b 2$, suggesting that the two proteins could interact (Figure 3C). As Asb2 is known to contain a SOCS box capable of interacting with Elongins and $\mathrm{Cul} 2 / 5$, it raises a possibility that Jak2 ubiquitination is mediated by ECS ligase complexes. In contrast, endogenous $\mathrm{Cul} 3$ and $\mathrm{Cul} 4$ were not brought down with Asb2 despite that they were readily detectable (Figure 3A). Even when they were co-transfected with Asb2, little

A P: antiFlag

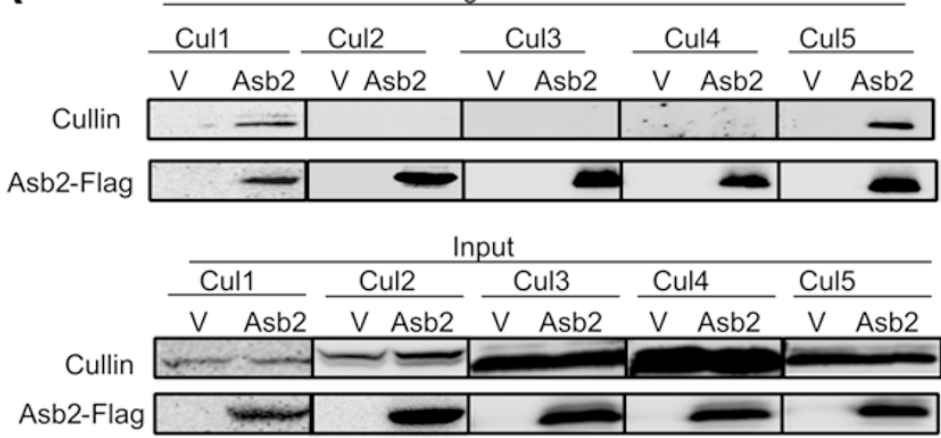

B

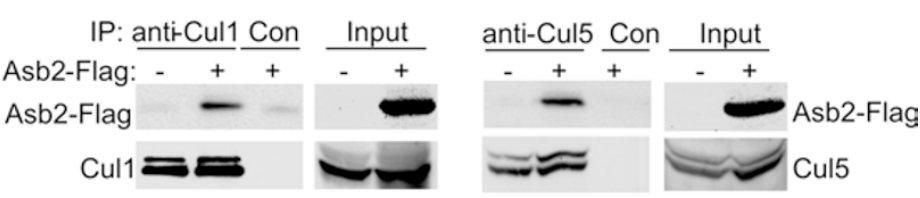

C

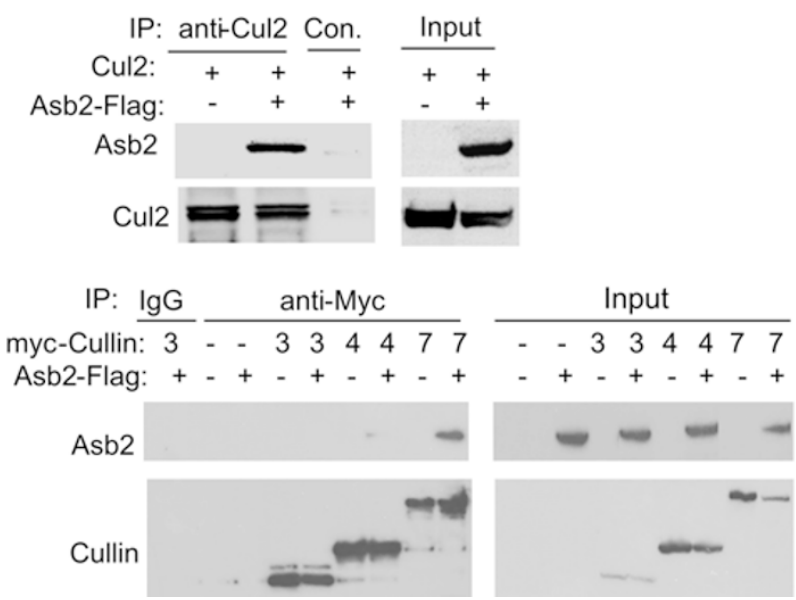

Figure 3 Interaction of Asb2 with Cullins. (A) Whole-cell lysates of vector or Asb2-Flag-transfected 293T cells were used in IP assays with an anti-Flag antibody in a lysis buffer containing $0.5 \%$ NP40. The precipitates as well as the input controls were immunoblotted with antibodies against indicated proteins. (B) 293T cells were transfected with or without Asb2-Flag. IP was performed with anti-Cul1 or Cul5 antibodies or control IgG. The precipitates were probed for indicated proteins. (C) $293 \mathrm{~T}$ cells were co-transfected with indicated cullin constructs, Cul2 or myc-tagged Cul3, 4 and 7, plus or minus Asb2. Cullins were pulled down with antibodies against Cul2 or the myc tag and the precipitates were analyzed for Asb2 and Cullins. 
interaction was detected (Figure 3C). This is consistent with our data that Notch and Asb2 had no effect on the turnover of a Cul3 substrate, NRF2 (data not shown) [29, 30]. Incidentally, when Asb2-Flag and myc-Cul7 were co-expressed, the anti-myc antibody could bring down Asb2, suggesting a possible interaction. Together, these results suggest that Asb2 has the potential to promote the ubiquitination of E47 and Jak2 by interacting with their respective cullin-based E3 ligases.

\section{Asb2 and SOCS1 bind Jak2 and Elongins in a mutually exclusive manner}

Jak2 is known to be bound to SOCS proteins, which are substrate-binding subunits of the ECS ligase complexes containing ElonginB and C, as well as Cul2/5 [12, 31-33]. Since Asb2 also contains a SOCS box, it was important to determine whether the binding of Asb2 and SOCS proteins to Jak2 are cooperative or mutually exclusive. Both Asb2 and SOCS1 but not SOCS3 interacted efficiently with Jak2 (Figure 4A and data not shown). When increasing amounts of SOCS1 were cotransfected with a constant amount of Asb2, the levels of Asb2 pulled down with anti-Jak2 antibodies decreased, suggesting that Asb2 and SOCS1 interact with Jak2 in a mutually exclusive manner (Figure 4A).

To determine whether Asb2 competes with SOCS1 to associate with Elongin B and $\mathrm{C}$, we introduced a constant amount of SOCS1 and increasing amounts of Asb2 together with constructs expressing myc-His-tagged ElonginB and ElonginC (Figure 4B). The amount of SOCS1 co-precipitated with Elongin B and $\mathrm{C}$ decreased as the amount of Asb2 increased, indicating that the two proteins compete to interact with the Elongins. However, unlike the strong interaction between Asb2 and Jak2 in both NP40 and RIPA buffers, Asb2 associates with Cul5 or Elongins only in the NP40 but not in RIPA buffer (Figure 4C and 4D). Taken together, these results suggest that Asb2 is able to displace SOCS proteins to form complexes with ElonginB and $\mathrm{C}$, as well as Cul5.

\section{Dissection of the functional domains in Asb2}

To better understand the function of Asb2, we mapped the domains mediating the interaction between Asb2 and substrates or components of the E3 ligases using coIP assays. Asb2 contains ankyrin repeats and a SOCS box. We initially tested constructs lacking the SOCS box or the ankyrin repeats $(\Delta \mathrm{C}$ and $\Delta \mathrm{N}$, respectively). Additional deletion mutants were then generated to further dissect the interacting domains (Figure 5A). Both $\Delta \mathrm{C}$ and $\Delta \mathrm{N}$ mutants interacted with Jak2 efficiently in RIPA buffer, suggesting that the essential interaction sequence lies between amino acids 419 and 528 (Figure 5B). We next showed that the N-terminal 328 amino acids were unable to bind Jak2, but internal deletions of amino acids 406-434 and 329-483 did not affect the interaction (Figure $5 \mathrm{~B})$. Thus, the Jak2 interacting domain of Asb2 was narrowed down from amino acids 483 to 528 .

Structural studies have indicated that the SOCS box directly contacts ElonginC, which is in a complex with ElonginB and Cul2/5 [34-36]. The SOCS box also contains residues responsible for $\mathrm{Cul} 5$ interaction. Therefore, it is not surprising that the $\triangle \mathrm{C}$ mutant lacking the SOCS box is unable to co-precipitate endogenous $\mathrm{Cul} 5$ or mycHis-tagged ElonginB (Figure 5C). On the other hand, the $\Delta \mathrm{N}$ mutant containing the SOCS box but lacking ankyrin repeats is capable of associating with both Elongins and Cul5 (Figure 5C). More interestingly, the association between Elongins and Cul5 was significantly strengthened by the SOCS box in Asb2 because co-transfection with full-length or $\Delta \mathrm{N}$ but not $\Delta \mathrm{C}$ facilitated the co-precipitation of $\mathrm{Cul} 5$ with antibodies against His-tagged ElonginB (Figure 5D).

Since Asb2 associates with Cul1, we examined the interaction between Asb2 and other components of the Cul1-containing $\mathrm{SCF}^{\mathrm{Skp} 2}$ E3 ligase complex. Skp2 exhibited a strong binding to Asb2. Since the $\Delta \mathrm{C}$ but not $\Delta \mathrm{N}$ mutant had similar binding activity as the full-length protein, it is likely that Skp2 binding is independent of the SOCS box (Figure 5E). Furthermore, the Skp2 interacting domain is localized primarily to the region between amino acids 328 and 406 , as the $\Delta \mathrm{N}, \Delta 328$ and $\Delta 328 / 483$ mutants showed no or poor interaction whereas the $\Delta 406 / 434$ mutant retained binding activity (Figure 5E). Collectively, results from these analyses suggest that Asb2 possesses separate domains responsible for interaction with Skp2, Jak2 and ElonginB/C/Cul5 (Figure 5A).

\section{Relationship among Skp2, Asb2 and substrates}

We then want to determine whether Asb2 associates with E47 through its interaction with Skp2. As shown in Figure 6A, Asb2 was co-transfected with or without E47 plus or minus Skp2 shRNA [6]. IP followed by immunoblotting analysis revealed that expression of the shRNA against Skp2 dramatically reduces the amount of E47 brought down with Asb2. Conversely, overexpression of Skp2 along with Asb2 and E47 significantly increased the amount of E47 co-precipitated with Asb2 (Figure $6 \mathrm{~B})$. Hence, these results suggest that the interaction between E47 and Asb2 is mediated through Skp2.

To examine the role of Skp2 in the interaction between Asb2 and Jak2, we knocked down Skp2 and evaluated the ability of Jak2 to associate with Asb2. Unlike the situation in the interaction between E47 and Asb2, Jak2 and Asb2 formed complexes equally well in the pres- 
A

\begin{tabular}{cccccccc}
\cline { 3 - 6 } Asb2-Flag $(\mu \mathrm{g}):$ & - & - & 5 & 5 & 5 & 5 & 5 \\
C4-SOCS1 $(\mu \mathrm{g}):$ & - & 5 & - & 2.5 & 5 & 7.5 & 5
\end{tabular}

Asb2-Flag

HPC4-SOCS1

Jak2

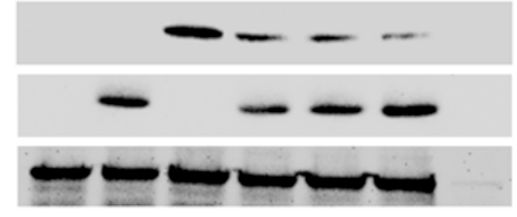

B

IP:

anti-myc Con

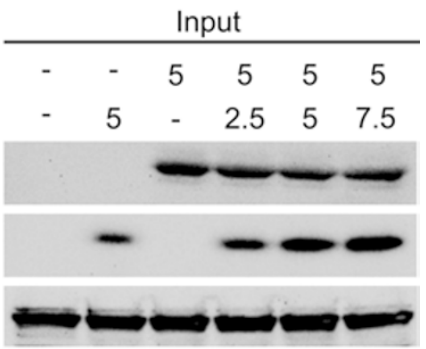

\begin{tabular}{|c|c|c|c|c|c|c|}
\hline \multicolumn{7}{|c|}{ Myc-His-Elongin B+Elongin C } \\
\hline- & - & 1 & 2.5 & 5 & 5 & 5 \\
\hline- & 5 & 5 & 5 & 5 & - & 5 \\
\hline
\end{tabular}

Asb2-Flag $(\mu \mathrm{g})$

HPC4-SOCS1 $(\mu \mathrm{g})$

Asb2-Flag

HPC4-SOCS1

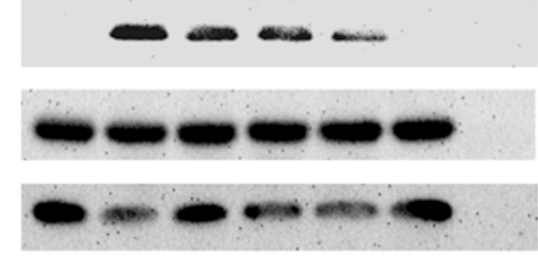

Myc-His-ElonginB

ElonginC

C

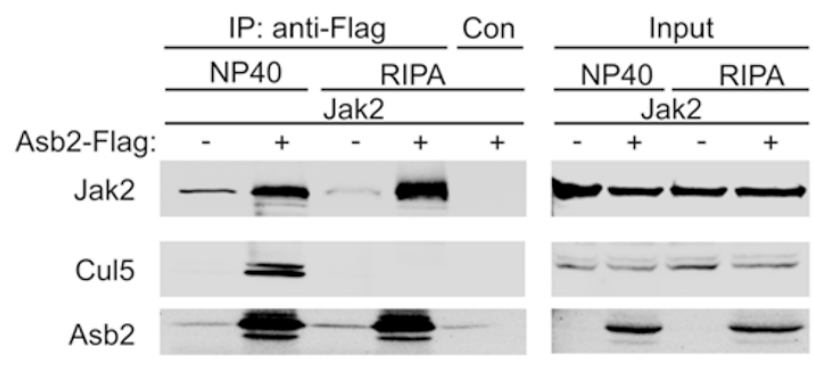

D

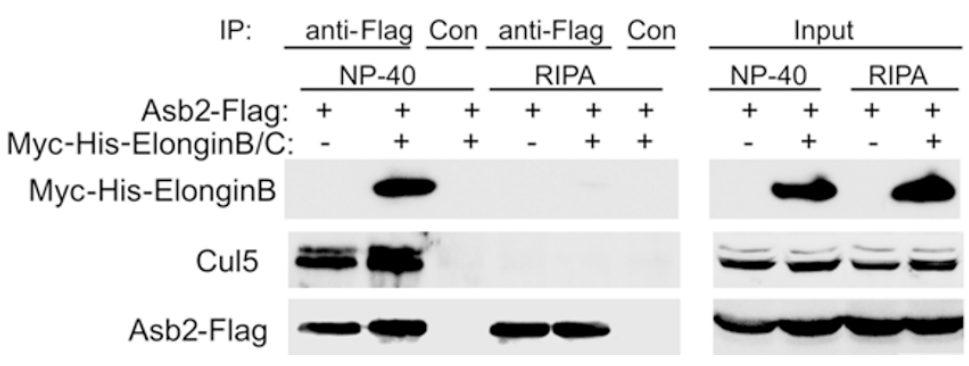

Figure 4 Interaction of Asb2 with components of Cul5-based E3 ligase complexes. (A) Asb2 competes with SOCS1 to bind to Jak2. 293T cells were co-transfected with indicated amounts of indicated constructs. IP was performed with anti-Jak2 or control IgG (Con) in the NP40 lysis buffer. The immunoprecipitates and inputs were immunoblotted with antibodies against the Flag or HPC4 tag and Jak2. (B) Asb2 competes with SOCS1 to bind to ElonginB/C. 293T cells were co-transfected with indicated amounts of indicated constructs. IP was performed with an anti-myc antibody or control IgG in the NP40 lysis buffer. The anti-Myc immunoprecipitates were subjected to immunoblotting with antibodies against the Flag or HPC4 tag or Elongin proteins. (C, D) Asb2 interacts more strongly with Jak2 than with ElonginB/C or Cul5. 293T cells were transfected with indicated constructs. IP was carried out in either the NP40 or RIPA buffer with indicated antibodies. The precipitates were analyzed by probing for the indicated proteins. 
A
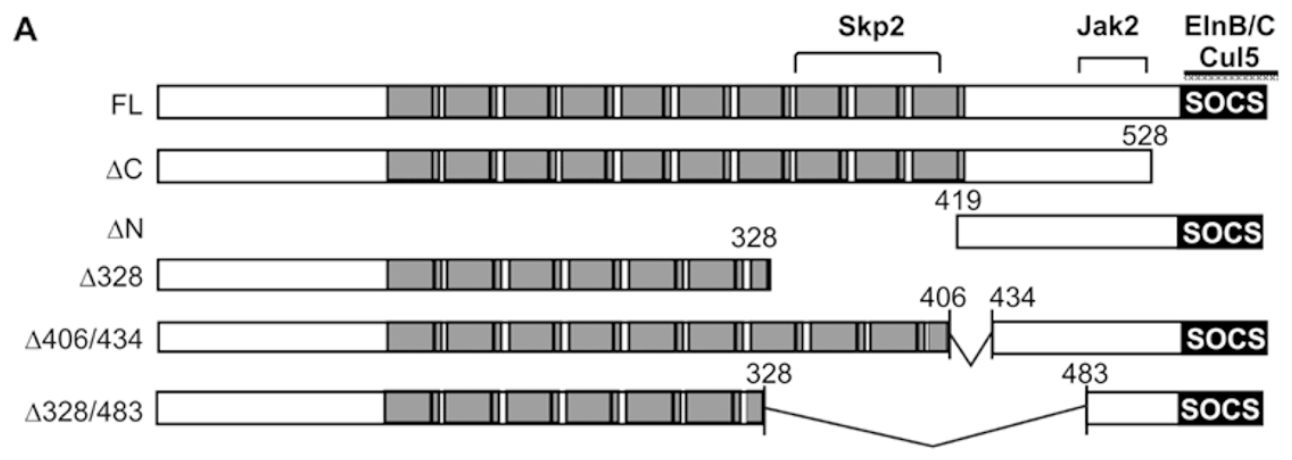

B

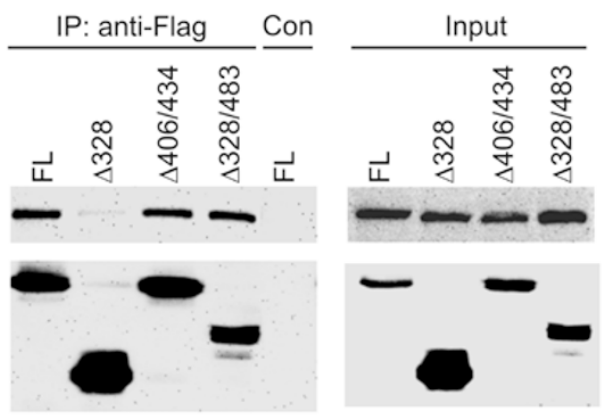

C

$\frac{\text { IP: anti-Flag }}{\text { Myc-His-Elongin } \mathrm{B}+\mathrm{C}} \frac{\text { Con }}{\text { Myc-His-Elongin } \mathrm{B}+\mathrm{C}}$

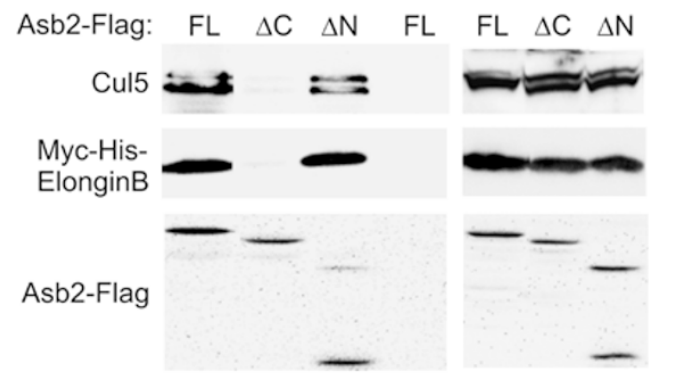

D $\quad \frac{\text { IP: Anti-His }}{\text { Myc-His-Elongin } \mathrm{B}+\mathrm{C}} \frac{\text { Con }}{\text { Myc-His-Elongin } \mathrm{B}+\mathrm{C}}$
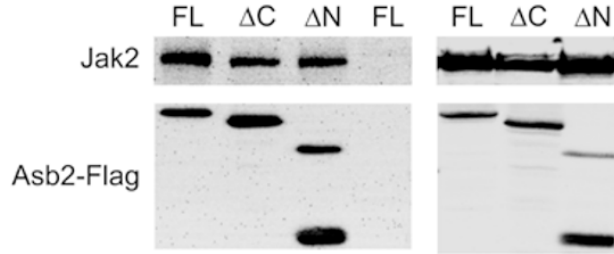

Asb2-Flag:

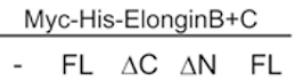

Cul5
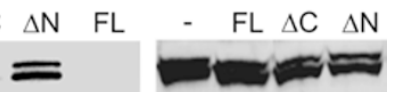

E
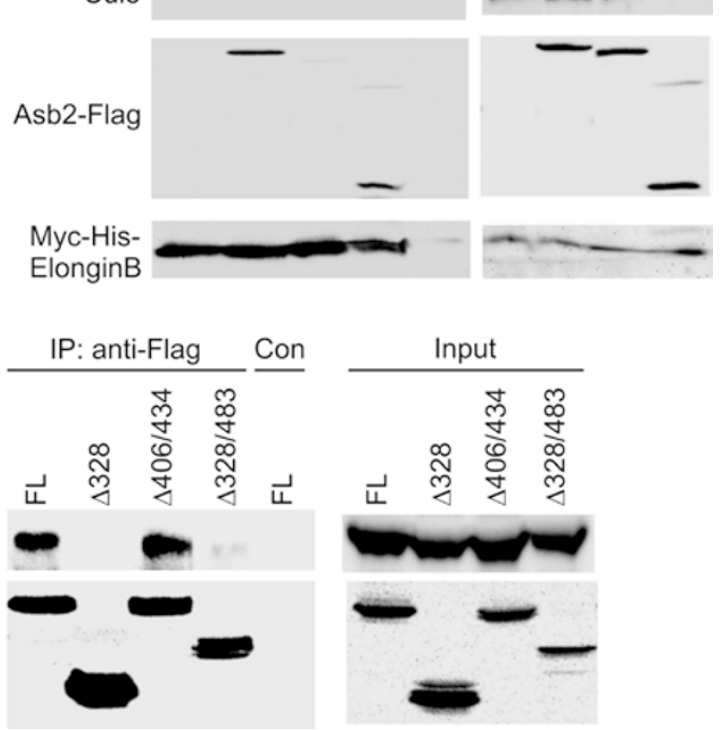

Figure 5 Mapping protein interacting domains of Asb2. (A) Schematic diagram of human Asb2 and its mutant constructs. Ankyrin repeats are marked as gray boxes and the black box represents the SOCS box. Numbers indicate the positions in amino acid sequence of Asb2. Internal deletions are designated by " $\mathrm{v}$ " shaped lines. All constructs contain a Flag tag at the C terminus. Regions responsible for Skp2, Jak2 and ElonginB/C/Cul5 binding are sketched on top of the full-length construct. (B) Interaction between Asb2 and Jak2. Jak2 was co-transfected with indicated constructs into 293T cells. IP was performed with anti-Flag or control antibodies in the RIPA buffer. The precipitates and inputs were probed with anti-Jak2 and anti-Flag antibodies. (C, D) Interaction between Asb2 and ElonginB/C or Cul5. 293T cells were co-transfected with indicated constructs. IP was carried out with anti-Flag (C) or anti-His (D) antibodies in the NP40 lysis buffer. IP with control IgG was included in each of the experiments. The precipitates were analyzed by immunoblotting with anti-Cul5, anti-Myc and anti-Flag antibodies. (E) Interaction of Asb2 with Skp2. Skp2 was co-transfected with indicated constructs. Anti-Flag or control immunoprecipitates obtained in the RIPA buffer were subjected to immunoblotting with anti-Skp2 and anti-Flag antibodies. 
ence or absence of Skp2 shRNA, suggesting that Jak2 and Asb2 form complexes independently of Skp2 (lanes 4 and 5, Figure 6C). This observation is consistent with data shown in Figures 3 and 4, where the interaction between Jak2 and Asb2 was readily detectable without co-expression of Skp2. However, co-expression of Skp2 significantly increased the amount of Asb2 brought down with Jak2, suggesting that Skp2 facilitates the interaction between Jak2 and Asb2 (lane 2 and 3, Figure 6C). On the other hand, the association between either endogenous

\begin{tabular}{|c|c|c|c|c|c|}
\hline A & IP: & anti- & & Con & Input \\
\hline E47: & - & + & + & + & -++ \\
\hline Asb2-Flag: & + & + & + & + & $+\quad+$ \\
\hline shRNA: & - & S & C & S & - S C \\
\hline E47 & & & $\cdots$ & $6=$ & $-\cdots$ \\
\hline Skp2 & & & isived & $3 \%$ & $-\div$ \\
\hline sb2- & & & & & - \\
\hline
\end{tabular}

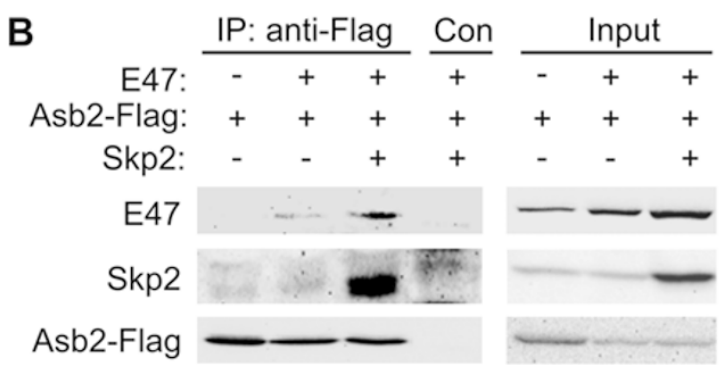
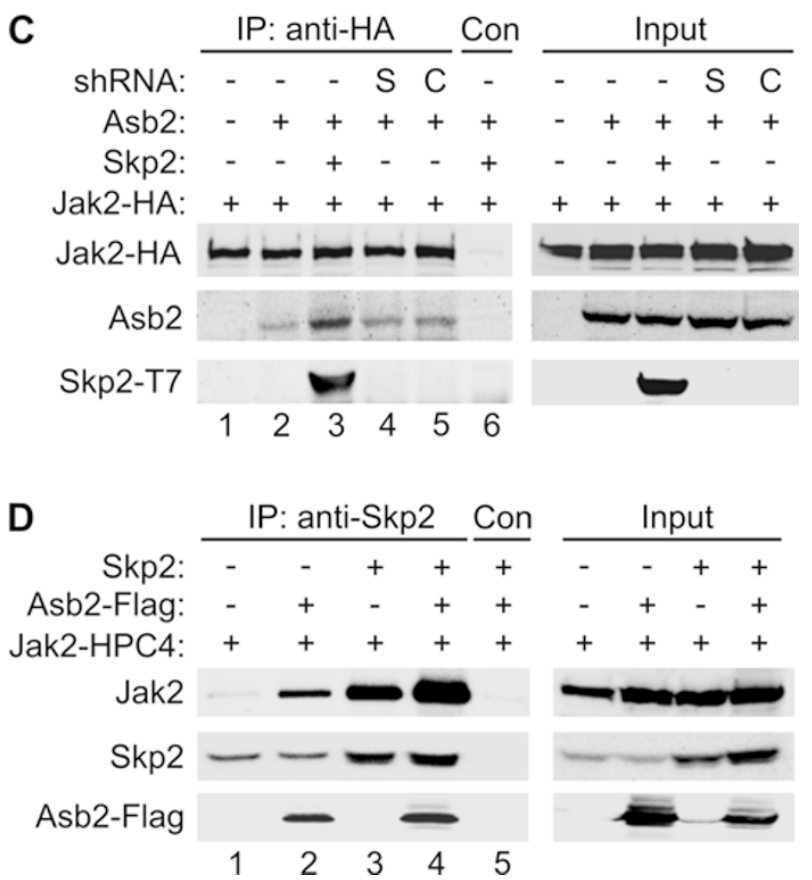

or exogenous Skp2 with Jak2 was also enhanced by coexpression of Asb2 (comparing lanes 1 vs 2 or lanes 3 vs 4, Figure 6D). Therefore, it appears that Jak2, Asb2 and Skp2 interact with each other to form a stable complex.

\section{Skp2 and Asb2 bridge the formation of a Cul1-Cul5 di-} meric E3 ligase complex

Because Skp2 and Asb2 interacted efficiently with each other and are able to associate with Cul1- and Cul5based E3 ligase complexes, respectively, it is possible that Skp2 and Asb2 could unite Cull and Cul5 plus their associated subunits to form a $\mathrm{Cul1} / \mathrm{Cul} 5$ dimeric E3 ligase complex. To explore this possibility, we first determined the dependence of Asb2-Cul1 interaction on Skp2 using co-IP assays. Both full-length Asb2 and the $\Delta \mathrm{C}$ mutant were able to co-precipitate with endogenous Cul1 (Figure 7A). However, the $\Delta \mathrm{N}$ mutant, which could not interact with Skp2, did not bring down Cul1. Furthermore, knockdown of Skp2 abolished the interaction between Asb2 and Cul1 (Figure 7A). Thus, it appears that Asb2 associates with Cul1 through Skp2.

Next, we examined the role of Asb2 in the Skp2Cul5 interaction (Figure 7B). Expression of Asb2 clearly increased the amount of Cul5 brought down by IP with anti-Skp2 antibodies in the NP40 buffer. The low level of Cul5 co-precipitated in the absence of exogenous Asb2 could be mediated by endogenous Asb2 or other Asb proteins. In line with the fact that Asb2 is incapable of interacting with Cul5 in the RIPA buffer (Figure 4D), the association between Skp2 and Cul5 was eliminated under this stringent condition (Figure 7B). In contrast, Skp2 coprecipitated with Cull in both NP40 and RIPA buffers.

Figure 6 Interaction of Asb2 and Skp2 with substrates. (A) Downregulation of Skp2 diminishes the association of Asb2 with E47. Constructs expressing E47 and Asb2-Flag were cotransfected into 293T cells with or without shRNA constructs against Skp2 (S) or a random control sequence (C). The antiFlag or control immunoprecipitates along with inputs were immunoblotted for the indicated proteins. (B) Skp2 facilitates the interaction between Asb2 and E47. Indicated constructs were co-transfected into 293T cells. IP and immunoblotting were performed as in A. (C) Asb2 and Jak2 interaction is independent of but strengthened by Skp2. Asb2-Flag and Jak2-HA were coexpressed with or without Skp2-T7 expressing or anti-Skp2 shRNA constructs in 293T cells. IP was performed with the anti$\mathrm{HA}$ and control antibodies in the NP40 lysis buffer. The amounts of Asb2-Flag and Skp2-T7 co-precipitated were determined by immunoblotting with antibodies against the indicated proteins. (D) Skp2 and Jak2 interaction is enhanced by Asb2. Indicated constructs were transfected into 293T cells. Immunoprecipitates of anti-Skp2 or control antibodies obtained in RIPA buffer were probed for the indicated proteins. 
A
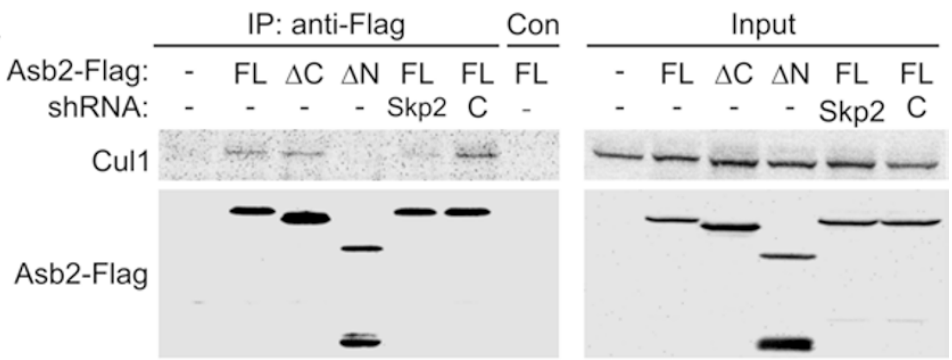

B

NP40 Buffer

RIPA Buffer

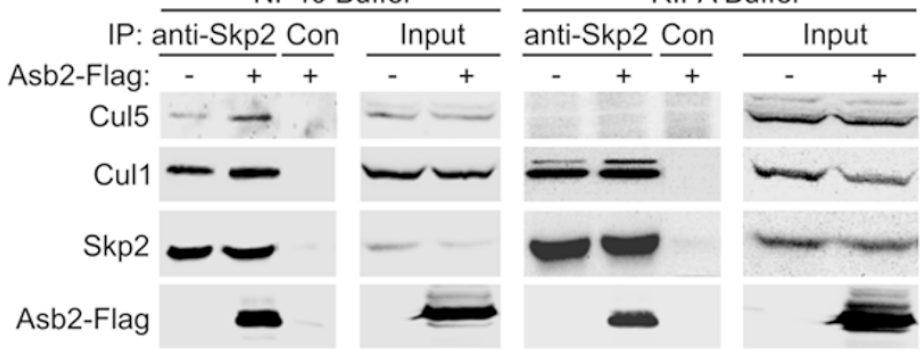

C

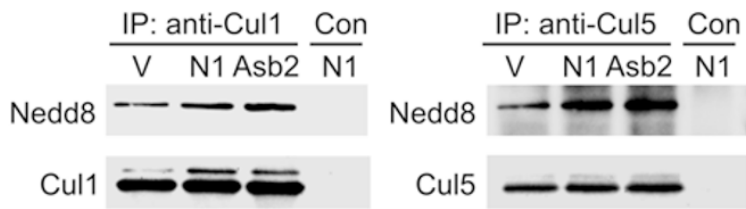

D

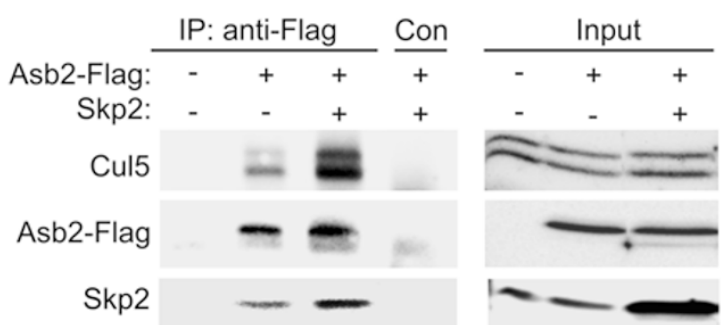

Figure 7 Asb2 and Skp2 bridge the assembly of a Cul1- and Cul5-containing heterodimeric E3 ligase complex. (A) Association of Asb2 with Cul1 depends on Skp2. Constructs expressing full-length or deletion mutant Asb2 were co-transfected into 293 T cells with or without Skp2 or control shRNA constructs. The anti-Flag or control immunoprecipitates obtained in the NP40 lysis buffer were immunoblotted with anti-Cul1 and anti-Flag antibodies. (B) Skp2 interacts with Cul5 in the NP40 but not RIPA buffer. 293T cells were transfected with or without Asb2, and IP was performed with anti-Skp2 or control antibodies in the NP-40 lysis and RIPA buffer, respectively. The immunoprecipitates were probed for the indicated proteins. (C) N1-IC (N1) or Asb2 was transfected into 293T cells. IP was performed with anti-Cul1 or Cul5 antibodies or control. The precipitates were immunoblotted with anti-Nedd8 and Cul1 or Cul5 antibodies. (D) Augmentation of Cul5 and Asb2 interaction by Skp2. 293T cells were co-transfected with indicated constructs. The anti-Flag or control immunoprecipitates obtained in the NP40 buffer were subjected to immunoblotting with anti-Cul5, anti-Skp2 and anti-Flag antibodies.

The slower migrating band co-precipitated in the RIPA buffer may represent the neddylated form of Cul1, which is thought to be present in active E3 ligases. Incidentally, anti-Nedd8 immunoblotting of Cul1 and Cul5 immunoprecipitates obtained in the presence of de-neddylation inhibitors revealed an increase in the amounts of neddylated Cull and Cul5 in cells transfected with Asb2 as compared with vector controls (Figure 7C).

Furthermore, the effect of Skp2 on Asb2-Cul5 interaction was examined by transfecting Asb2 with or without 
Skp2 and performing co-IP assays. Expression of Skp2 significantly elevated the amount of endogenous Cul5 brought down by immunoprecipitating Asb2 (Figure 7D). Collectively, the data presented in Figure 7 suggest that the interaction between Skp2 and Asb2 offers an opportunity to join Cul1 and Cul5 together with their associated subunits, thus forming a non-canonical dimeric E3 ligase complex with two catalytic centers, which could be more efficient at ubiquitin ligation.

Notch-or Asb2-induced E47 and Jak2 degradation depends on both Cull and Cul5

If Notch-induced Asb2 expression results in the formation of a Cul1-Cul5 dimeric E3 ligase complex, which in turn efficiently catalyzes ubiquitination of either E47 or Jak2, one would expect that downregulation of either Cul1 or Cul5 would rescue the degradation of these substrates. In contrast, if E47 and Jak2 were ubiquitinated by independent E3 ligases consisting of either Cul1 or Cul5, respectively, diminishing the function of either of the cullins would have distinct effects on Notch- or Asb2induced degradation of their corresponding substrates. To test these possibilities, we first utilized DN mutants of Cul1 and Cul5, which lack the C-terminus responsible for interacting with ring-finger proteins such as Rbxl or 2 and thus render the E3 ligase complexes catalytically inactive [37]. In co-transfection assays, the stimulatory effects of N1-IC and Asb2 on E47 or Jak2 degradation were abolished by co-expression of either DN Cull or Cul5 (Figure 8A). Consistent with these results, the ubiquitination of E47 and Jak2 enhanced by Asb2 was also dramatically diminished by either DN-Cul1 or DN-Cul5 (Figure 8B).

Finally, we utilized siRNAs against Cul1 and Cul5 to reduce the levels of these proteins in cells co-transfected with E47 or Jak2 with or without N1-IC or Asb2. Like the findings obtained by using DN mutants, knocking down either Cul1 or Cul5 significantly counteracted Notch- or Asb2-induced degradation of both E47 and Jak2 (Figure 8C). Hence, these results support the notion that Asb2 promotes the ubiquitination of both E47 and Jak2 by facilitating the formation of a dimeric complex consisting of both Cul1 and Cul5 along with their associated subunits.

\section{Discussion}

Asb2-mediated formation of non-canonical dimeric E3 ligase complexes

$\mathrm{SCF}^{\text {Skp2 }}$ and ECS E3 ligases have been thought to be responsible for the ubiquitination of their respective substrates, E47 and Jak2 [6, 12]. These canonical monomer- ic E3 ligase complexes might be involved in the steadystate turnover of E47 and Jak2, as they are present more or less ubiquitously or in situations where the substrates are ubiquitinated. For example, cytokine signaling leads to Jak2 activation by tyrosine phosphorylation and induction of SOCS gene expression, which then allows ubiquitination of phosphorylated Jak2 [38]. However, under special circumstances, these monomeric E3 ligases could be shifted to a heterodimeric form that may be catalytically more active. In this report, we provide an example of such a scenario, namely, Notch-induced Asb2 expression and Asb2-mediated formation of a non-canonical Cul1- and Cul5-containing ubiquitin ligase complex.

The interaction between Skp2 and Asb2 allows the association between Cul1- and Cul5-based E3 ligases. Cul1 and Cul5 serve as the docking sites for adaptors of Skp2 and Asb2 at their N-termini and for ring-finger proteins and E2 enzymes at their C-termini. Skp2 and Asb2 also serve as substrate acceptors and thus position the substrates between two sets of E3 ligases (Figure 9). This configuration has been proposed for homodimeric E3 ligases formed through the dimerization of substratebinding subunits or through the dimerization of Nedd8 attached on cullins [39-42]. Our findings illustrate for the first time that a heterodimeric complex could be formed through the association of two different proteins, which in turn brings together distinct sets of E3 ligase components. However, it should be noted that this model is based on in vivo protein-protein interaction data. In vitro assembly of this multi-subunit complex from purified components may or may not be possible depending on whether it requires more auxiliary proteins yet to be identified. The native forms of this complex have not been recovered from cells, pending the availability of suitable reagents.

Besides the accessibility of two catalytic centers to the substrates, an additional advantage the dimeric E3 ligase complexes can offer is the stabilization of neddylated forms of cullins, probably by preventing their deneddylation $[17,18]$. Indeed, we have found that levels of neddylated Cul1 and Cul5 were increased in the presence of Notch1 and Asb2 (Figure 7C). Effectively, more complexes could be kept in an active state rather than an inactive form, which is bound to the inhibitor, CAND1 $[19,20,43]$.

We have shown that Asb2 possesses separate domains that mediate its interaction with Skp2, Jak2 and Elong$\mathrm{inB} / \mathrm{C}$ plus Cul5. Likewise, Skp2 is expected to associate with Asb2, Skp1 and its various substrates, including E47. Given the extended structure of the leucine-rich repeats of Skp2, it is not difficult to envision Skp2 accommodating these multiple interfaces. Skp2 represents 

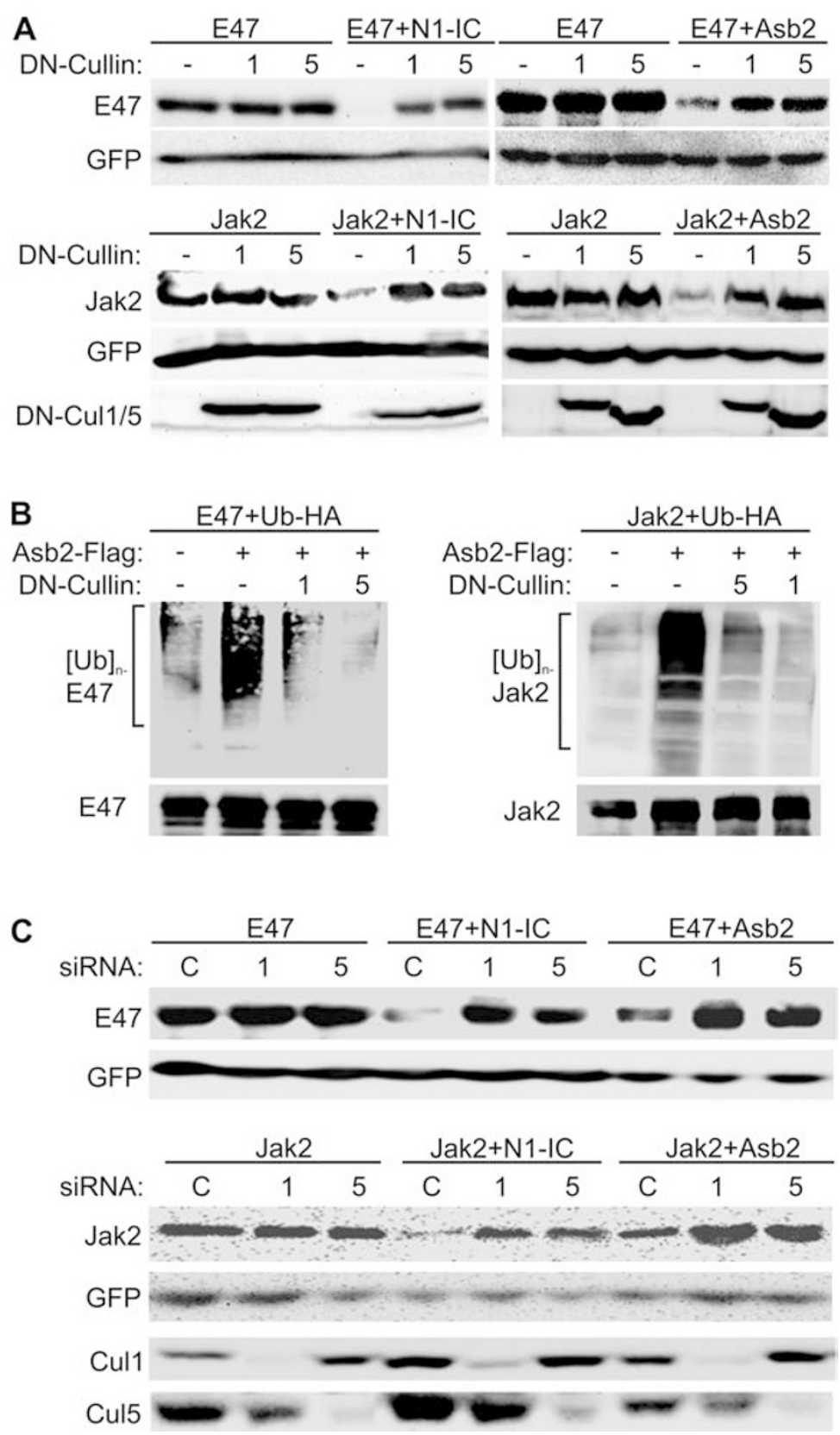

Figure 8 Dependence of both Cul1 and Cul5 in Notch and Asb2-induced degradation of E47 and Jak2. (A) N1-IC and Asb2induced degradation of E47 and Jak2 was attenuated by DN mutant-Cul1 (1) or Cul5 (5). E47 and GFP constructs were cotransfected with or without N1-IC or Asb2 plus or minus DN-Cul1 or DN-Cul5 into NIH3T3 cells. Jak2, GFP and EpoR constructs were co-transfected with or without N1-IC or Asb2 plus or minus DN-Cul1 or DN-Cul5 into 293T cells and treated with $5 \mathrm{ng} / \mathrm{ml}$ of Epo for $1.5 \mathrm{~h}$ prior to harvesting the cells. Immunoblot assays were performed with antibodies against the indicated proteins. (B) Abrogation of Asb2-induced ubiquitination of E47 and Jak2 by either DN-Cul1 or DN-Cul5. 293T cells were cotransfected with the indicated constructs. IP was carried out with anti-E47 or Jak2 antibodies after transfected cells were treated with MG-132 at a concentration of $5 \mu \mathrm{M}$ for $5 \mathrm{~h}$. The precipitates were subjected to immunoblotting with the anti-HA monoclonal antibody to visualize ubiquitinated E47 and Jak2, respectively. The amounts of E47 and Jak2 precipitated were determined by probing with anti-E47 and Jak2 antibodies. (C) Downregulation of either Cul1 or Cul5 rescues both N1-IC and Asb2-induced protein degradation. HeLa cells were co-transfected with E47 or Jak2-HPC4 plus GFP-expressing constructs with or without N1-IC or Asb2. After $24 \mathrm{~h}$, the cells were then reverse transfected with $10 \mathrm{nM}$ of control or siRNAs targeting Cul1 or Cul5 using Lipofectamine 2000 for $12 \mathrm{~h}$ and incubated for additional $24 \mathrm{~h}$ in culture medium. Whole-cell lysates were immunoblotted with antibodies against the indicated proteins. 


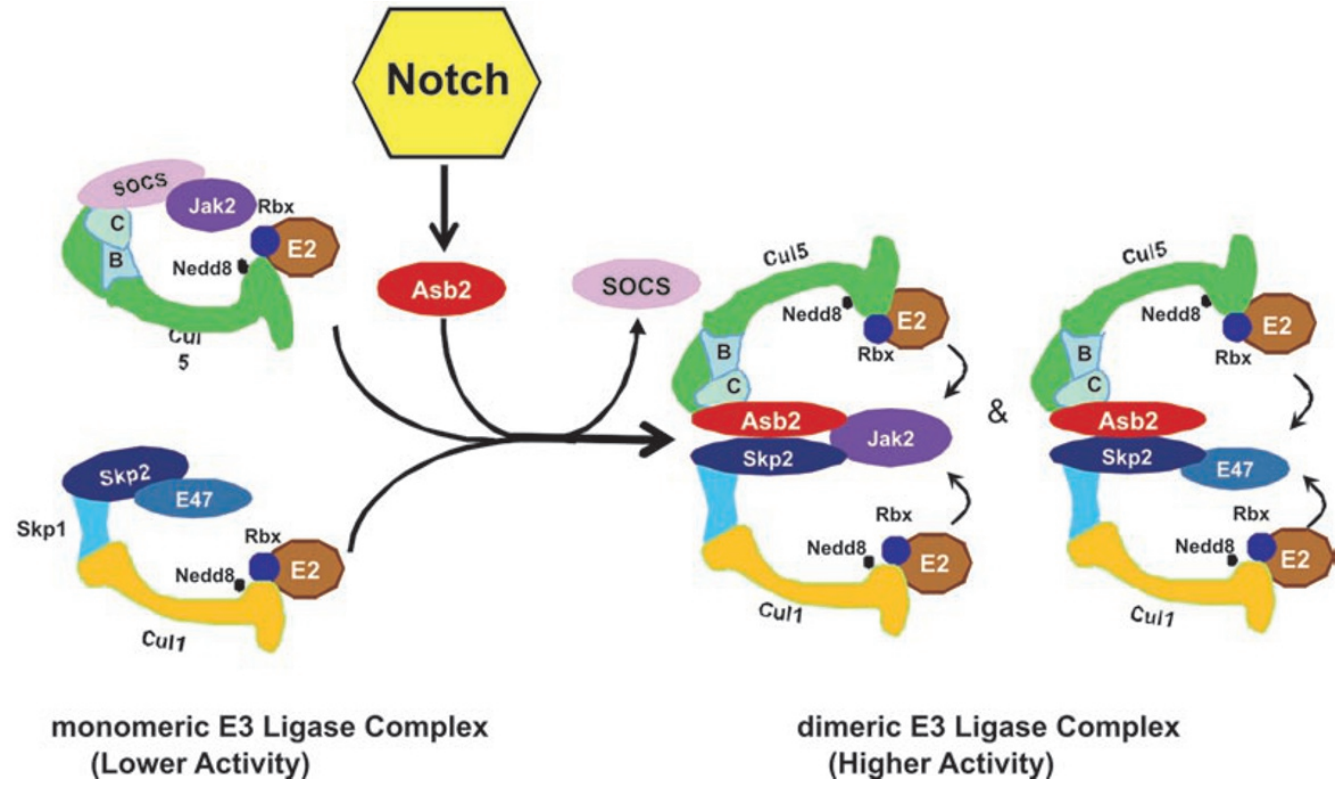

Figure 9 Asb2-mediated dimeric cullin-based complexes. Canonical Cul1 or Cul5 monomeric E3 ligase complexes are thought to be responsible for ubiquitination of their respective substrates. Activation of Notch signaling upregulates Asb2 expression, and Asb2 displace SOCS proteins to associate with ElonginB/C and Cul5. Interaction between Asb2 and Skp2 bridges the formation a dimeric complex containing Cul1 and Cul5 and their associates. The manner in which E47 and Jak2 interact with Asb2 and Skp2 is illustrated separately. The enhancement of ubiquitination by the availability of two sets of E2 enzymes to catalyze ubiquitin ligation is illustrated by arrows.

a family of 70 or so F-box proteins $[44,45]$. It would be interesting to determine whether Asb2 can interact with other F-box proteins. If so, the target pool of Asb2 would be greatly expanded, which could be highly significant for explaining the diverse biological effects of Notch signaling pathways.

\section{Roles of the Asb family in protein ubiquitination}

Asb2 belongs to a large family with 18 members in mouse and humans [14]. Like the Notch receptors, Asb homologs are also present in nematodes and insects. These Asb proteins all have a SOCS box at the $\mathrm{C}$-terminus and varying numbers of ankyrin repeats. Therefore, it is reasonable to suspect a certain degree of functional redundancy among the members. Indeed, we found that Asb1 behaved similarly to Asb2 in promoting E47 and Jak2 degradation (Figure 2C). However, Asb12 had little effects on the turnover of these proteins. This difference may be due to the divergence in their SOCS boxes. Whereas Asb1 and Asb2 share extensive sequence similarities in their SOCS boxes, the sequence of Asb12 SOCS box is quite different. Consistent with the inability of Asb12 to stimulate E47 and Jak2 degradation, Asb12 was found to bind poorly to Elongins, thus underscoring the importance of Asb-Elongin interaction for E47 and Jak2 ubiquitination (data not shown).
Furthermore, Asb3, Asb6 and Asb9 have also been shown to bind to Elongins and Cul5 to promote the ubiquitination of tumor necrosis factor receptor 2 (TNFR2), APS and creatine kinase B, respectively [22, 23, 46]. Similar to the recruitment of Jak2 by Asb2, these Asb proteins also associate with their respective substrates and it will be interesting to determine whether these Asb proteins can interact with F-box proteins. If they do, it is plausible that the ubiquitination of TNFR2, APS and CKB also involves heterodimeric E3 ligases containing Cul1 and Cul5.

Although Asb proteins all have multiple copies of ankyrin repeats, the function of these ankyrin repeats in the context of protein ubiquitination remains unclear. For example, Asb2 interacts with Jak2 and Elongins/ Cul5 with sequences outside the ankyrin repeats. The interacting domain for Skp2 overlaps with the C-terminal portion of the repeats. However, the Asb2 $\triangle 328$ construct could not bind to Skp2 despite the presence of six copies of ankyrin repeats, which suggests that the interaction is not mediated by the ankyrin repeats per se. Likewise, the association between Asb3 and TNFR2 is also independent of ankyrin repeats. Nevertheless, given the conservation of this well-known protein-protein interaction motif in all Asb proteins, ankyrin repeats are expected to play an important role, perhaps by interacting with addi- 
tional unknown proteins that may play important roles in the Asb-mediated assembly of multimeric complexes.

\section{Regulation of Asb gene expression}

Notch signaling has a profound impact on the turnover of several pivotal regulators of cell differentiation and proliferation [6-8, 47, 48]. Notch-induced Asb2 transcription can lead to the conversion of monomeric E3 ligases into heterodimeric E3 ligase complexes, which may be more effective at ubiquitin ligation. This provides Notch signaling with a powerful tool in regulating diverse biological processes by controlling protein stability. Since the ICs of Notch receptors act as transcriptional coactivators, it is likely that they stimulate transcription of the Asb2 gene through their DNA-binding partner, CSL, binding sites of which have been found in the regulatory sequences of the Asb2 gene. Considering that the large family of $A s b$ genes may arise through gene duplication events, the regulatory sequences may also be copied along with the coding sequences, thus preserving the regulatory mechanisms such as Notch-induced transcription. Indeed, we have found that expression of several $A s b$ genes besides Asb2 can also be stimulated by Notch signaling in a cell type-specific manner (data not shown). Likewise, levels of Notch-induced Asb2 expression also vary from one cell type to another. These observations would suggest that tissue-specific regulation of Asb gene expression can influence the effect of Notch signaling by independently or cooperatively controlling Asb gene transcription. In fact, the $A s b$ genes have been shown to have differential patterns of expression $[46,49]$.

On the other hand, Asb gene expression can also be augmented by additional signals independent of those originating from Notch receptors. For example, cytokine or Toll-like receptor signaling might significantly influence Asb gene expression, which in turn can promote protein ubiquitination and degradation. Expression of the $A s b 2$ gene has also been shown to be stimulated by retinoic acid $[49,50]$. Taking into account the potential of Asb proteins in mediating the ubiquitination of diverse substrates, upregulating transcription of the $A s b$ genes might be a critical mechanism for controlling various aspects of developmental biology and tumor biology. Therefore, it will be extremely important to investigate the transcriptional regulation of $A s b$ genes. Equally significant will be the expression profile of Asb proteins in pathological situations such as cancer and autoimmunity. With the accumulation of knowledge about Asb proteins, this previously under-appreciated family of proteins may emerge to be crucial regulators of many cellular processes.

\section{Materials and Methods}

Antibodies and chemicals

Rabbit polyclonal antibodies against $\mathrm{Cul1}, \mathrm{Cul2}, \mathrm{Cul} 3, \mathrm{Cul} 4$, Cul5, Skp2, Erk1, E2A.E12 and tubulin, and monoclonal antibodies against GFP were obtained from Santa Cruz Biotechnology. Monoclonal antibodies against $\mathrm{Cul1}, 6 \times$ His and SOCS1 were purchased from BD Transduction Laboratories. Anti-Flag monoclonal antibody (M2) was obtained from Sigma. Monoclonal antibodies against Cul5 and Skp2 were obtained from Invitrogen. Rabbit antiJak2 and anti-Asb2 antibodies were from Upstate Biotechnology and Imgenex, respectively. Rabbit anti-ElonginB and ElonginC antibodies were from Biolegend. Anti-Nedd8 and anti-Ubiquitin were from ZyMed. Monoclonal anti-HPC4 antibody was kindly provided by Dr Charles Esmon (Oklahoma Medical Research Foundation). Anti-HA (12CA5), anti-Myc (10E9) and anti-N1IC (bTAN20) were produced from hybridoma cell lines. Lipofectamine 2000 was purchased from Invitrogen. $N$-ethylmaleimide (NEM), iodoacetamide (IAA), MG-132 and GSI XIV were from Calbiochem. Recombinant mouse erythropoietin (Epo), IL-7, SCF and Flt3-L were obtained from R \& D Systems.

\section{Plasmids}

Human $3 \times$ Flag-Asb2, Asb2 $\Delta \mathrm{C}$, Asb1 and Asb12 were kindly provided by Dr Junya Kohroki (Tokyo University of Science, Japan). HPC4-tagged Asb2 retroviral construct was constructed by transferring the full-length of Asb2 from the $3 \times$ flag vector to the MIGR1 retroviral vector containing a HPC4 tag. Asb2 $\Delta \mathrm{N}$ was created by PCR amplification of desired fragment using $3 \times$ Flag-Asb2 as a template. Asb2 deletion constructs were created by restriction digestion at appropriate sites and ligation into the pcDNA3 vector. HPC4-SOCS1 construct was generated by PCR and cloned into pcDNA3. Mouse HA-EpoR/pcDAN3 was a gift of Dr Lily Huang (UT Southwest). HA-Jak2 was kindly supplied by Dr Olli Silvennoinen (University of Helsinki, Finland). HA-Cul1 and Myc-Cul2 constructs were generously offered by Dr Yue Xiong (UNC Medical School). Myc-His-Elongin B and HSV-ElonginC were courteously provided by Dr Joan Conaway (Stower Institute). Flag-DNCul1, Cul5, DN-Cul5, $\beta$-TrCP, Fbxw7 and FbxL6 constructs were obtained from Addgene. Mouse Jak2/pcDNA3 was graciously provided by Dr Zhizhuang J Zhao (University of Oklahoma Health Sciences Center) and Dr Martin Myers Jr (University of Michigan). N1-IC /pcDNA3, N1-IC/MIGR1, Skp2/pcDNA3 and Skp2T7/pcDNA3, as well as constructs expressing Skp2 and control shRNA, were as previously described $[6,47]$.

\section{Transfection}

NIH3T3, 293T and HeLa cells were co-transfected using the calcium phosphate method. To introduce siRNA into HeLa cells transfected with appropriate DNA constructs, $10 \mathrm{nM} \mathrm{Cul1,} \mathrm{Cul5}$ or control siRNAs were reverse-transfected $24 \mathrm{~h}$ later using Lipofectamine 2000 according to manufacturer's instruction (Invitrogen). Human Cull and Cul5 siRNA oligonucleotides (Cull: GGAUGAGAGUGUACUGAAAtt; Cul5: GGCAUACUUGGAUUCAACAtt) and a negative control siRNA oligonucleotides were purchased from Ambion. Immunoblot analyses were performed at $36 \mathrm{~h}$ post siRNA transfection. 
IP and immunoblotting assay

Transiently transfected $293 \mathrm{~T}$ cells with the indicated expression constructs were fed with new culture medium for $1.5 \mathrm{~h}$ before harvest, and the cells were lysed in either $0.5 \% \mathrm{NP}-40$ lysis buffer or RIPA buffer containing a cocktail of protease inhibitors, $1 \mathrm{mM}$ $\mathrm{NaF}, 100 \mu \mathrm{M} \mathrm{Na}_{3} \mathrm{VO}_{4}$ and $5 \mu \mathrm{M} \mathrm{MG}-132$. For probing neddylated cullins, the IP buffer also contains $4 \mathrm{mM}$ NEM and $5 \mathrm{mM}$ IAA. IP was usually done with $5 \mu \mathrm{g}$ of antibodies. Immunoprecipitates were analyzed using 8-12\% SDS-PAGE followed by immunoblotting with appropriate antibodies.

\section{Ubiquitination in vivo}

For analysis of the endogenous E2A and Jak2 ubiquination, $\mathrm{BaF} 3$ cells were transduced with N1-IC, Asb2 or GFP control retroviruses and were sorted as described [7]. Cells were IL-3 starved for $4 \mathrm{~h}$ and then re-stimulated with fresh culture medium containing 20\% WEHI-3 supernatant and 10\% FBS in the presence of 5 $\mu \mathrm{M}$ MG-132 for $5 \mathrm{~h}$. For detection of the in vivo ubiquitination levels of ectopic-expressed E47 and Jak2, 293T cells were cotransfected with $10 \mu \mathrm{g}$ of HA-tagged ubiquitin along with appropriate constructs followed by the treatment with $5 \mu \mathrm{M} \mathrm{MG}-132$ for $5 \mathrm{~h}$. Treated cells were lysed in $0.5 \% \mathrm{NP}-40$ lysis buffer containing protease inhibitors and $5 \mu \mathrm{M} \mathrm{MG}-132$. IP was performed with anti-E47 or anti-Jak2 antibodies. The ubiquitinated E2A and Jak2 in the precipitates were immunoblotted with monoclonal antiubiquitin or anti-HA antibodies.

\section{Quantitative real-time PCR}

Total RNAs were isolated using TRIzol and reverse transcribed using M-MLV reverse transcriptase (Invitrogen). Real-time PCR reactions were performed using Power SYBR Green PCR master Mix (Applied Biosystems) and primers for mouse Asb2 from Qiagen (catalog number QT00119889). Levels of Asb2 were normalized with those of $\beta$-actin.

\section{Acknowledgment}

We would like to thank Drs Yue Xiong, Joan Conaway, Martin Myers Jr, Zhizhuang J Zhao, Hui Zhang and Zhen-Qiang Pan for reagents and advice. This work was supported by grants from the NIH to X-HS (AI56129). X-HS holds the Lew and Myra Ward Chair in Biomedical Research.

\section{References}

1 Simpson P. Introduction: Notch signalling and choice of cell fates in development. Semin Cell Dev Biol 1998; 9:581-582.

2 Artavanis-Tsakonas S, Rand MD, Lake RJ. Notch signaling: cell fate control and signal integration in development. Science 1999; 284:770-776.

3 Tamura K, Taniguchi Y, Minoguchi S, et al. Physical interaction between a novel domain of the receptor Notch and the transcription factor RBP-J kappa/Su(H). Curr Biol 1995; 5:1416-1423.

4 Jarriault S, Brou C, Logeat F, et al. Signalling downstream of activated mammalian Notch. Nature 1995; 377:355-358.

5 Wu L, Aster JC, Blacklow SC, et al. MAML1, a human homologue of Drosophila mastermind, is a transcriptional co- activator for NOTCH receptors. Nat Genet 2000; 26:484-489.

6 Nie L, Xu M, Vladimirova A, Sun XH. Notch-induced E2A ubiquitination and degradation are controlled by MAP kinase activities. EMBO J 2003; 22:5780-5792.

7 Nie L, Perry SS, Zhao Y, et al. Regulation of lymphocyte development by cell-type-specific interpretation of notch signals. Mol Cell Biol 2008; 28:2078-2090.

8 Nie L, Wu H, Sun XH. Ubiquitination and degradation of Tal1/SCL are induced by notch signaling and depend on Skp2 and CHIP. J Biol Chem 2008; 283:684-692.

9 Kee BL. E and ID proteins branch out. Nat Rev Immunol 2009; 9:175-184.

10 Jackson PK, Eldridge AG. The SCF ubiquitin ligase: an extended look. Mol Cell 2002; 9:923-925.

11 Zheng N, Schulman BA, Song L, et al. Structure of the Cul1Rbx1-Skp1-F boxSkp2 SCF ubiquitin ligase complex. Nature 2002; 416:703-709.

12 Ungureanu D, Saharinen P, Junttila I, Hilton DJ, Silvennoinen O. Regulation of Jak2 through the ubiquitin-proteasome pathway involves phosphorylation of Jak2 on Y1007 and interaction with SOCS-1. Mol Cell Biol 2002; 22:3316-3326.

13 Kamura T, Sato S, Haque D, et al. The Elongin BC complex interacts with the conserved SOCS-box motif present in members of the SOCS, ras, WD-40 repeat, and ankyrin repeat families. Genes Dev 1998; 12:3872-3881.

14 Hilton DJ, Richardson RT, Alexander WS, et al. Twenty proteins containing a C-terminal SOCS box form five structural classes. Proc Natl Acad Sci USA 1998; 95:114-119.

15 Petroski MD and Deshaies RJ. Function and regulation of cullin-RING ubiquitin ligases. Nat Rev Mol Cell Biol 2005; 6:9-20.

16 Pickart CM. Mechanisms underlying ubiquitination. Annu Rev Biochem 2001; 70:503-533.

17 Ohh M, Kim WY, Moslehi JJ, et al. An intact NEDD8 pathway is required for Cullin-dependent ubiquitylation in mammalian cells. EMBO Rep 2002; 3:177-182.

18 Pan ZQ, Kentsis A, Dias DC, Yamoah K, Wu K. Nedd8 on cullin: building an expressway to protein destruction. Oncogene 2004; 23:1985-1997.

19 Zheng J, Yang X, Harrell JM, et al. CAND1 binds to unneddylated CUL1 and regulates the formation of SCF ubiquitin E3 ligase complex. Mol Cell 2002; 10:1519-1526.

20 Liu J, Furukawa M, Matsumoto T, Xiong Y. NEDD8 modification of CUL1 dissociates p120(CAND1), an inhibitor of CUL1-SKP1 binding and SCF ligases. Mol Cell 2002; 10:1511-1518.

21 Heuze ML, Guibal FC, Banks CA, et al. ASB2 is an Elongin $\mathrm{BC}$-interacting protein that can assemble with Cullin 5 and Rbx1 to reconstitute an E3 ubiquitin ligase complex. J Biol Chem 2005; 280:5468-5474.

22 Chung AS, Guan YJ, Yuan ZL, Albina JE, Chin YE. Ankyrin repeat and SOCS box 3 (ASB3) mediates ubiquitination and degradation of tumor necrosis factor receptor II. Mol Cell Biol 2005; 25:4716-4726.

23 Debrincat MA, Zhang JG, Willson TA, et al. Ankyrin repeat and suppressors of cytokine signaling box protein asb-9 targets creatine kinase B for degradation. J Biol Chem 2007; 282:4728-4737.

24 Kohroki J, Nishiyama T, Nakamura T, Masuho Y. ASB pro- 
teins interact with Cullin5 and Rbx2 to form E3 ubiquitin ligase complexes. FEBS Lett 2005; 579:6796-6802.

25 Mahrour N, Redwine WB, Florens L, et al. Characterization of Cullin-box sequences that direct recruitment of Cul2-Rbx1 and Cul5-Rbx2 modules to Elongin BC-based ubiquitin ligases. J Biol Chem 2008; 283:8005-8013.

26 Kamizono S, Hanada T, Yasukawa H, et al. The SOCS box of SOCS-1 accelerates ubiquitin-dependent proteolysis of TELJAK2. J Biol Chem 2001; 276:12530-12538.

27 Sarmento LM, Huang H, Limon A, et al. Notch1 modulates timing of G1-S progression by inducing SKP2 transcription and p27 Kip1 degradation. J Exp Med 2005; 202:157-168.

28 Pajerowski AG, Nguyen C, Aghajanian H, Shapiro MJ, Shapiro VS. NKAP is a transcriptional repressor of notch signaling and is required for T cell development. Immunity 2009; 30:696-707.

29 Cullinan SB, Gordan JD, Jin J, Harper JW, Diehl JA. The Keap1-BTB protein is an adaptor that bridges Nrf2 to a Cul3based E3 ligase: oxidative stress sensing by a Cul3-Keap1 ligase. Mol Cell Biol 2004; 24:8477-8486.

30 Furukawa M, Xiong Y. BTB protein Keap1 targets antioxidant transcription factor Nrf2 for ubiquitination by the Cullin 3-Roc1 ligase. Mol Cell Biol 2005; 25:162-171.

31 Bergamin E, Wu J, Hubbard SR. Structural basis for phosphotyrosine recognition by suppressor of cytokine signaling-3. Structure 2006; 14:1285-1292.

32 Waiboci LW, Ahmed CM, Mujtaba MG, et al. Both the suppressor of cytokine signaling 1 (SOCS-1) kinase inhibitory region and SOCS-1 mimetic bind to JAK2 autophosphorylation site: implications for the development of a SOCS-1 antagonist. J Immunol 2007; 178:5058-5068.

33 Frantsve J, Schwaller J, Sternberg DW, Kutok J, Gilliland DG. Socs-1 inhibits TEL-JAK2-mediated transformation of hematopoietic cells through inhibition of JAK2 kinase activity and induction of proteasome-mediated degradation. Mol Cell Biol 2001; 21:3547-3557.

34 Bullock AN, Rodriguez MC, Debreczeni JE, Songyang Z, Knapp S. Structure of the SOCS4-ElonginB/C complex reveals a distinct SOCS box interface and the molecular basis for SOCS-dependent EGFR degradation. Structure 2007; 15:1493-1504.

35 Bullock AN, Debreczeni JE, Edwards AM, Sundstrom M, Knapp S. Crystal structure of the SOCS2-elongin C-elongin B complex defines a prototypical SOCS box ubiquitin ligase. Proc Natl Acad Sci USA 2006; 103:7637-7642.

36 Babon JJ, Sabo JK, Soetopo A, et al. The SOCS box domain of SOCS3: structure and interaction with the elonginBCcullin5 ubiquitin ligase. J Mol Biol 2008; 381:928-940.
37 Jin J, Ang XL, Shirogane T, Wade HJ. Identification of substrates for F-box proteins. Methods Enzymol 2005; 399:287309.

38 Wormald S, Hilton DJ. Inhibitors of cytokine signal transduction. J Biol Chem 2004; 279:821-824.

39 Wimuttisuk W, Singer JD. The Cullin3 ubiquitin ligase functions as a Nedd8-bound heterodimer. Mol Biol Cell 2007; 18:899-909.

40 Zimmerman ES, Schulman BA, Zheng N. Structural assembly of cullin-RING ubiquitin ligase complexes. Curr Opin Struct Biol 2010 Sep 27. doi:10.1016/j.sbi.2010.08.010

41 Bosu DR, Kipreos ET. Cullin-RING ubiquitin ligases: global regulation and activation cycles. Cell Div 2008; 3:7.

42 Merlet J, Burger J, Gomes JE, Pintard L. Regulation of cullinRING E3 ubiquitin-ligases by neddylation and dimerization. Cell Mol Life Sci 2009; 66:1924-1938.

43 Siergiejuk E, Scott DC, Schulman BA, et al. Cullin neddylation and substrate-adaptors counteract SCF inhibition by the CAND1-like protein Lag2 in Saccharomyces cerevisiae. EMBO J 2009; 28:3845-3856.

44 Jin J, Cardozo T, Lovering RC, et al. Systematic analysis and nomenclature of mammalian F-box proteins. Genes Dev 2004; 18:2573-2580.

45 Frescas D, Pagano M. Deregulated proteolysis by the F-box proteins SKP2 and beta-TrCP: tipping the scales of cancer. Nat Rev Cancer 2008; 8:438-449.

46 Wilcox A, Katsanakis KD, Bheda F, Pillay TS. Asb6, an adipocyte-specific ankyrin and SOCS box protein, interacts with APS to enable recruitment of elongins $\mathrm{B}$ and $\mathrm{C}$ to the insulin receptor signaling complex. J Biol Chem 2004; 279:3888138888.

47 Huang Z, Nie L, Xu M, Sun XH. Notch-induced E2A degradation requires CHIP and Hsc70 as novel facilitators of ubiquitination. Mol Cell Biol 2004; 24:8951-8962.

48 Smith EM, Akerblad P, Kadesch T, Axelson H, Sigvardsson M. Inhibition of EBF function by active Notch signaling reveals a novel regulatory pathway in early B-cell development. Blood 2005; 106:1995-2001.

49 Kohroki J, Fujita S, Itoh N, et al. ATRA-regulated $A s b$-2 gene induced in differentiation of HL-60 leukemia cells. FEBS Lett 2001; 505:223-228.

50 Guibal FC, Moog-Lutz C, Smolewski P, et al. ASB-2 inhibits growth and promotes commitment in myeloid leukemia cells. J Biol Chem 2002; 277:218-224.

51 Bookout AL, Mangelsdorf DJ. Quantitative real-time PCR protocol for analysis of nuclear receptor signaling pathways. Nucl Recept Signal 2003; 1:e012. 